\title{
Einstein Probe - a small mission to monitor and explore the dynamic X-ray Universe
}

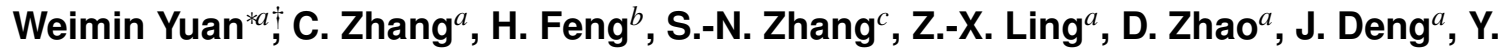 \\ Qiu $^{a}$, J.P. Osborne ${ }^{d}$, P. O’Brien ${ }^{d}$, R. Willingale ${ }^{d}$, J. Lapington ${ }^{d}$, G.W. Fraser ${ }^{d} ;$ \& the \\ Einstein Probe team
}

a Key Laboratory of Space Astronomy and Technology, National Astronomical Observatories

(NAOC), Chinese Academy of Sciences, Datun Rd 20A, Beijing 100012 China

b Department of Engineering Physics, Tsinghua University, Beijing 100084, China

c Institute of High Energy Physics (IHEP), Chinese Academy of Sciences, 100049, Beijing, China

$d$ Department of Physics and Astronomy, University of Leicester, Leicester, LE1 7RH, UK

\begin{abstract}
Einstein Probe is a small mission dedicated to time-domain high-energy astrophysics. Its primary goals are to discover high-energy transients and to monitor variable objects in the $0.5-4 \mathrm{keV} \mathrm{X-}$ rays, at higher sensitivity by one order of magnitude than those of the ones currently in orbit. Its wide-field imaging capability, featuring a large instantaneous field-of-view $\left(60^{\circ} \times 60^{\circ}, \sim 1.1 \mathrm{sr}\right)$, is achieved by using established technology of micro-pore (MPO) lobster-eye optics, thereby offering unprecedentedly high sensitivity and large Grasp (effective area times field-of-view). To complement this powerful monitoring ability, it also carries a narrow-field, sensitive followup X-ray telescope based on the same MPO technology to perform follow-up observations of newly-discovered transients. Public transient alerts will be downlinked rapidly, so as to trigger multi-wavelength follow-up observations from the world-wide community. Over three of its 97minute orbits almost the entire night sky will be sampled, with cadences ranging from 5 to 25 times per day. The scientific objectives of the mission are: to discover otherwise quiescent black holes over all astrophysical mass scales by detecting their rare X-ray transient flares, particularly tidal disruption of stars by massive black holes at galactic centers; to detect and precisely locate the electromagnetic sources of gravitational-wave transients; to carry out systematic surveys of Xray transients and characterise the variability of X-ray sources, such as high-redshift gamma-ray bursts, supernova shock breakouts, X-ray binaries of compact objects, gamma-ray bursts, active galactic nuclei and stellar coronal flares, etc. Einstein Probe has been selected as a candidate mission of priority (no further selection needed) in the Space Science Programme of the Chinese Academy of Sciences, aiming for launch around 2020.
\end{abstract}

Swift: 10 Years of Discovery,

2-5 December 2014

La Sapienza University, Rome, Italy

\footnotetext{
*Speaker.

†E-mail:wmy@nao.cas.cn

†Deceased, March 2014
} 


\section{Introduction}

Transients and variable objects pervade the X-ray Universe; some indeed are the most energetic outbursts in the Universe. Observations and interpretations of these phenomena have revolutionised our understanding of the Universe and its underlying physical laws. They provide otherwise inaccessible laboratories to explore the limits of contemporary physics and to study matter under extreme conditions. A rich variety of transients and variables, with diverse timescales from sub-seconds to years, have been discovered and extensively studied since the early days of X-ray astronomy, thanks to successive all-sky monitors in the X-rays and $\gamma$-rays. This exciting field of discovery is highlighted by Swift [1], which has made many remarkable and surprising discoveries during the past 10-year's operation, from short gamma-ray bursts (GBRs) and high-redshift GRBs, to supernova shock breakouts, magnetars, and relativistic tidal disruption events, etc. [2]. More discoveries continue to be made in recent years with the joining of Fermi [3] and MAXI [4] in the game.

Despite rapid progress in this field, many key questions remain unanswered, and yet more have been introduced. New phenomena continue to be discovered and appeal for observations on a large scale for characterisation, others are highly expected and await discovery. Particularly, with the advent of major facilities across the electromagnetic spectrum and in the multi-messenger realms of gravitational wave and neutrinos, time-domain astronomy will enter a golden era towards the end of this decade. This makes monitoring and exploration of the X-ray sky even more exciting, and calls for the next generation of wide-field monitoring instruments with higher sensitivity so as to extend the horizons of the monitored Universe significantly beyond the reach of the ones currently in orbit.

Driven by the great scientific potential, the Einstein Probe (EP) ${ }^{1}$ mission was proposed by the Chinese Academy of Sciences (CAS) institutes (NAOC \& IHEP) and Tsinghua University in 2012, in response to a call of the CAS for small- and medium-sized candidate missions in its newly initiated space science programme. In 2015, EP has been selected to be among three candidate missions of priority aiming for launch around 2020, and is funded for advanced study by the CAS under its Strategic Priority Research Programme of Space Science. The project is open for international participation via collaboration, which currently involves mainly the UK.

\section{Scientific Objectives}

Are black holes prevalent in our Universe and how does matter fall onto them? What kind of electromagnetic radiation and sources are associated with cosmic gravitational-wave events, and where and how are they both produced? When and where did the first generation of stars form and how did they re-ionize the dark early Universe? What happens at the very first instance when a star explodes and generates a supernova? EP will try to address these questions by capturing faint flashes of X-ray radiation produced by energetic events within a cosmic horizon far beyond the reach of any current and previous missions. Its primary scientific objectives are:

(1) Reveal quiescent black holes at almost all astrophysical mass scales and study how matter falls onto them by detecting transient X-ray flares, particularly stars being tidally-disrupted by otherwise

\footnotetext{
${ }^{1}$ http://ep.bao.ac.cn
} 
dormant massive black holes at galactic centres.

(2) Discover the X-ray photonic counterparts of gravitational-wave transients found with the next generation of gravitational-wave detectors and precisely locate them.

(3) Carry out systematic and sensitive surveys of high-energy transients, to discover faint X-ray transients, such as high-redshift GRBs, supernova shock breakout, and new types of transients.

\subsection{Discover and explore otherwise quiescent black holes via catching X-ray flares}

A star approaching a massive black hole (MBH) closely enough will be tidally disrupted and accreted when its self-gravity cannot balance the strong tidal force, producing a flare of electromagnetic radiation [5]. First discovered in the ROSAT survey [6] and later from archival multiwavelength surveys, the majority of such tidal disruption events (TDEs) identified so far (about two dozen) were found via their luminous transient X-ray emission [7]. As perhaps the most unique signature of the existence of MBHs in otherwise quiescent galactic nuclei [5], TDEs provide a census of MBHs in the Universe, i.e. to constrain their occupation fractions in various types and masses of galaxies, which is essential for understanding the formation and evolution of galaxies and MBHs. Furthermore, observations of the detailed processes of these violent events would provide otherwise inaccessible laboratories to explore how, and what happens when, matter falls on to a black hole from start to end. Despite that only a handful events are known so far and mostly were found in the declining phase with sparsely sampled data, a rich variety of the demography and physical processes involved have been revealed. Recent discoveries of a few jetted events by Swift and MAXI indicated that relativistic jets can be launched in at least some of the TDEs [8]. A distinctive X-ray lightcurve with large drops and recurrences was also seen in one case, which was interpreted as perhaps the most accessible signature of quiescent binary MBHs, via tidally disrupting a star [9]. Theoretical modeling also predicts that the effects of General Relativity may be tested, and the black hole spins be measured, from the well-sampled X-ray lightcurves of TDEs [10]. Moreover, TDEs provide an effective way to discover intermediate-mass black holes (IMBHs) with masses of $10^{2-5} \mathrm{M}_{\odot}$ lurking at the centre of dwarf galaxies, which are otherwise hard to find in observations.

EP is an ideal instrument to search systematically for and study TDEs, and is expected to detect them at the peaks of their X-ray flares out to at least a few hundred Mpc. The estimated detection rate for EP may range from several tens to hundreds per year. The majority can be caught at the rising phase of the flares, making it possible to observe in multiple wavelengths the complete process of TDEs from the onset of disruption. EP will also be able to detect more relativistic TDEs with jets out to redshifts $z>1$, and more events with the possible signatures of binary MBHs. EP is expected to revolutionise the field of TDE research by detecting and characterising TDEs in large numbers with well-sampled data and catching them at an early flaring phase. This will greatly advance our understanding of the demography, formation and evolution of MBHs, as well as the physics of accretion and jet formation.

Moreover, EP will discover new stellar- and intermediate-mass black holes lurking in our Milky Way and in nearby galaxies, in the galactic plane or even at the centres of globular clusters, by detecting their X-ray outbursts due to some kind of instability of gas accretion.

\subsection{Detect and precisely locate electromagnetic sources of gravitational-wave transients}

Direct detection of the long-sought gravitational wave $(\mathrm{GW})$ signal is perhaps one of the most 
important events in modern physics and astrophysics, and will close the last missing link of the predictions of General Relativity. With the advent of the next generation of GW detectors (such as Advanced LIGO and Virgo), the detection of GW sources is highly anticipated towards the end of this decade. Those detectors are expected to detect mergers of binary neutron stars (NS) or a neutron star with a black hole, that are strong transient GW sources, out to several-hundred Mpc. The predicted combined detection rate, albeit with a considerable uncertainty, would be the order of $\sim 0.2-200 \mathrm{yr}^{-1}$ from 2019 [11]. However, GW sources are hard to locate, such that only 10-30\% will have position accuracies better than 20 square degrees. The detection of accompanying electromagnetic transients (EM counterparts) is essential for locating precisely and identifying associated astrophysical sources, and for measuring redshifts (thus the source distance and EM energy budget). These are important data for understanding the nature of the GW events. Therefore the EM counterparts are of great interest to both the GW and astrophysical communities. A potentially even more far-reaching application is in cosmology; joint detections of GW and EM events at high redshifts can be used to probe the geometry and expansion of the Universe, since GW events from binary mergers are the standard sirens from which the luminosity distances could be inferred independently [12], while the EM measurements lead to the redshifts.

NS-NS mergers are thought to be the origin of short GRBs, and simultaneous detection of an SGRB-like X-ray transient with a GW transient would provide the definitive confirmation of this model. In addition, recent models also predict that, in the case where a massive magnetar formed as an (intermediate) merger product, transient X-ray emission can be produced nearly isotropically on relatively short timescales up to hours [13]; the isotropy of the emission implies that the chance of being detected is significantly higher than that of highly beamed jet emission from short GRBs.

With the combined high sensitivity, large FoV, manoeuvrability and rapid alert downlink, EP is expected to be a major contributor to this exciting new field. By synergy with the next generation of GW detectors, EP is expected to either detect the photonic sources simultaneously with GW events, or rapidly observe the sky region covering the large error boxes provided by the GW detectors.

\subsection{Systematic surveys of X-ray transients}

EP will carry out systematic all-sky surveys to discover high-energy transients of various types over a wide range of timescales and at high cadence in the soft X-rays. It will also perform immediate follow-up observations of newly discovered transients with its narrow-field X-ray telescope onboard, and will issue fast alerts to trigger follow-up observations by multi-wavelength facilities world-wide. Of particular interest, EP aims at detecting more high-redshift GRBs and at higher redshifts than the currently known sample (7 GRBs with $z=6.2-9.4$ ). These valued events, produced by stars in the early Universe, carry unique information on early star-formation and metallicity evolution, and hopefully on the first generation of stars and the re-ionization in the dark early Universe, which are otherwise almost inaccessible from ground-based facilities. Shock breakout emission from supernovae is the prompt X-ray emission produced as the outward-propagating shocks generated by core-collapse breaks out of the stellar surface. X-ray observations can yield important clues to the properties of the progenitor stars. There are only a few candidate events known so far [14] due to their elusiveness given the short burst durations $\left(\sim 10^{3} \mathrm{~s}\right)$ and moderate brightness. EP is also expected to detect more of such events. Other transient sources to detect and characterise in large numbers include X-ray flashes, low-luminosity GRBs, X-ray rich GRBs, GRB precursors, 
Table 1: Specifications of WXT and FXT

\begin{tabular}{|c|c|c|}
\hline Parameters & WXT & FXT \\
\hline Number of modules & 8 & 1 \\
\hline Field-of-view & $60^{\circ} \times 60^{\circ}$ & $1^{\circ} \times 1^{\circ}$ \\
\hline Focal length (mm) & 375 & 1,400 \\
\hline Angular resolution FWHM (arcmin) & $<5$ & $<5$ \\
\hline Bandpass (keV) & $0.5-4.0$ & $0.5-4.0$ \\
\hline Energy resolution@1 keV & $40 \%$ & $100 \mathrm{eV}$ \\
\hline Effective area (central focus) $\left(\mathrm{cm}^{2}\right)$ & $3 @ 0.7 \mathrm{keV}$ & $60 @ 1 \mathrm{keV}$ \\
\hline Sensitivity (erg s${ }^{-1} \mathrm{~cm}^{-2} @ 1,000 \mathrm{~s}$ ) & $\sim 1 \times 10^{-11}$ & $\sim 3 \times 10^{-12}$ \\
\hline
\end{tabular}

magnetars, stellar corona flares, classical novae, supergiant fast X-ray transients, and outbursts of active galactic nuclei and blazars, etc.

\section{Instruments}

EP will carry two scientific instruments-a survey instrument Wide-field X-ray Telescope (WXT) with a large instantaneous Field-of-View $\left(\mathrm{FoV}, 60^{\circ} \times 60^{\circ}\right)$ and a narrow-field $\left(1^{\circ} \times 1^{\circ}\right)$ Follow-up X-ray Telescope (FXT), as well as a fast alert downlink system. To achieve both wide FoV and X-ray focusing, the novel Micro-Pore Optics (MPO) in the lobster-eye configuration [15][16] is adopted for WXT. The MPO lobster-eye optics is made of a thin spherical micro-channel plate with millions of square micro-pores, the axes of which all point radially to a common centre of curvature. Grazing incidence reflection from the sides of the pores can focus incoming X-rays onto a focal sphere with a radius of half the curvature of the optics. The point spread function, which is cruciform, has a central peaking spot produced by two reflections from adjacent walls of the pores and cross-arms produced by single reflections. Such an optics can yield a large focusing gain of $\sim 2000$, and a moderate resolution of FWHM the order of arc-minutes delivered by the currently available MPO pieces. In practice, the MPO Lobster-eye optics can achieve a FoV of thousands of square degrees with very light weight, which is unique for wide-field X-ray imaging.

WXT consists of eight modules with a $375 \mathrm{~mm}$ focal length (Figure 1, left panel). Modules 1-6 are identical; each has an aperture size of $280 \mathrm{~mm} \times 280 \mathrm{~mm}$ and is composed of $7 \times 7$ mosaicking MPO pieces, subtending $20^{\circ} \times 20^{\circ}$ square degrees. Modules 7 and 8 have an aperture size of $400 \mathrm{~mm} \times 280 \mathrm{~mm}$ each and subtend $30^{\circ} \times 20^{\circ}$ square degrees per module. The total FoV of WXT is $60^{\circ} \times 60^{\circ}(\sim 1.1$ steradian $)$. To match the large-format focal plane, gas detectors based on GEM (Gas Electron Multiplier) are under development, and Xenon gas will be used. The detectors have a size of $14 \times 14 \mathrm{~cm}^{2}$ for each of modules $1-6$, and $14 \times 20 \mathrm{~cm}^{2}$ for modules 7-8. Figure 1 (right panel) shows a prototype of the gas detectors of one WXT module for preliminary tests. The nominal bandpass of WXT is $0.5-4.0 \mathrm{keV}$.

Mounted at the centre of the payload, FXT adopts the same MPO focusing technology considering the simplicity of the technology and the relatively low budget of the mission. It has a much longer focal length of $1.4 \mathrm{~m}$, leading to a much larger effective area than that of WXT $\left(\sim 60 \mathrm{~cm}^{2}\right.$ 


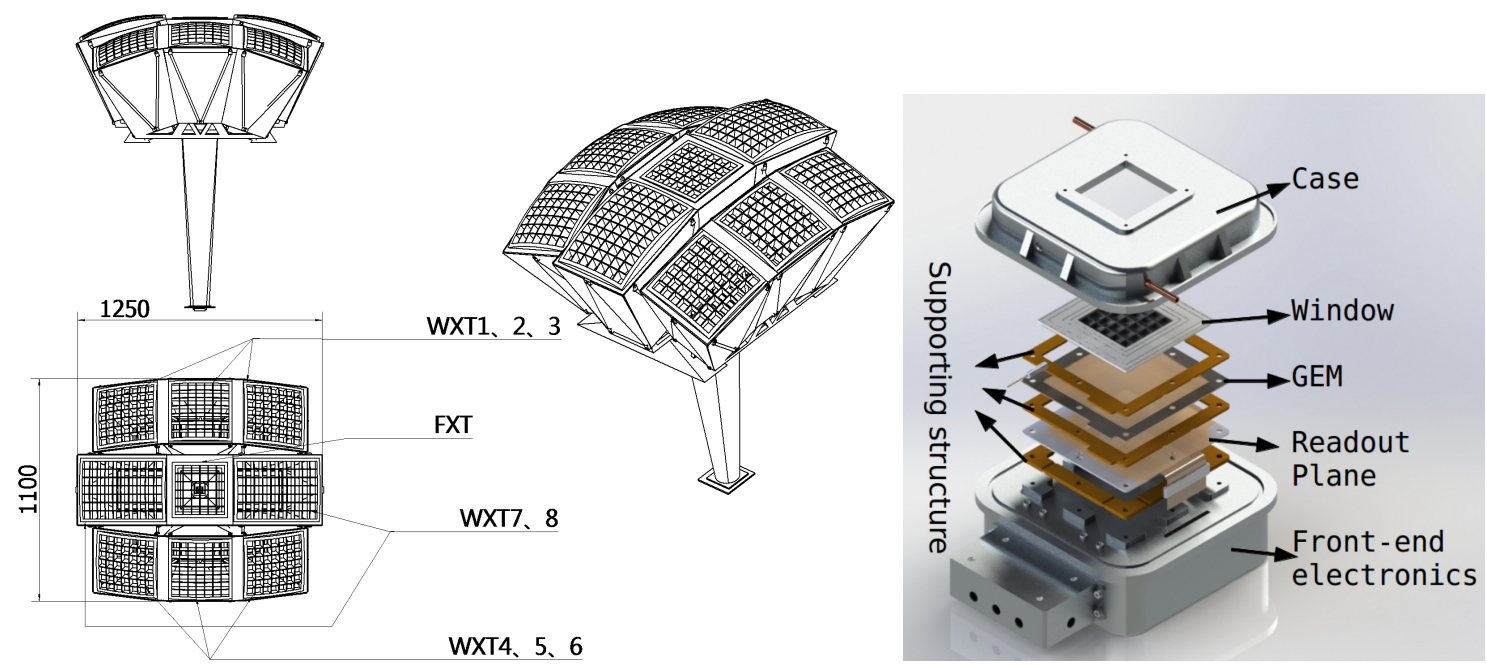

Figure 1: (left) Layout of WXT modules and FXT. (right) Sketch of a GEM-based gas detector as the focal plane detector for a WXT module.

@ $1 \mathrm{keV}$ ). FXT has an aperture size of about $240 \mathrm{~mm} \times 240 \mathrm{~mm}$, which is mosaicked by $6 \times 6 \mathrm{MPO}$ pieces. A CCD will be used as the focal plane detector of FXT to gain better spectral resolution.

The specifications of WXT and FXT are summarised in Table 1. Figure 2 (left panel) shows the effective area curves for WXT. These data are obtained via realistic ray-tracing simulations taking into account the imperfectness of the MPO arrays [17], which are in good agreement with the simulation results from the Q software developed at the University of Leicester [16]. The Grasp parameter (effective area times FoV) is also shown in Figure 2 (right panel).

\section{Mission Profile}

The combination of an array of wide-field lobster-eye modules to act as a soft X-ray transient monitor and a narrow-field lobster eye telescope for follow-up observations gives unprecedented grasp and sensitivity which will revolutionise X-ray transient astronomy. The payload has low weight $(150 \mathrm{~kg})$ and low power consumption $(200 \mathrm{~W})$, which can be easily accommodated by one of the micro or small satellite platforms readily available in China. One possible configuration is shown in Figure 3, which has the total weight of the satellite about $450 \mathrm{~kg}$ including the payload. The satellite will be in a circular orbit at an altitude of $\sim 600 \mathrm{~km}$ and a period of $\sim 97$ minutes, and an inclination angle of $30^{\circ}$ or less.

The survey strategy of EP will be composed of a series of pointings to mosaic the night sky in the directions avoiding the Sun. During each 97-minute orbit of the satellite, five fields will be observed on the night-side of the sky, each with an 11-minute exposure. Over three orbits almost the entire night sky would be sampled, with cadences ranging from 5 to 25 times per day. Alternative schemes of e.g. $\sim 20$ minutes exposure for each pointing are also under consideration. The pointing directions are shifted by about 1 degree per day to compensate the daily movement of the Sun on the sky. In this way, the entire sky will be covered within half-a-year's operation.

Once a transient source is detected with WXT and is classified and triggered by the processing and alerting system onboard, the satellite will slew to a new position to enable pointed follow-up 

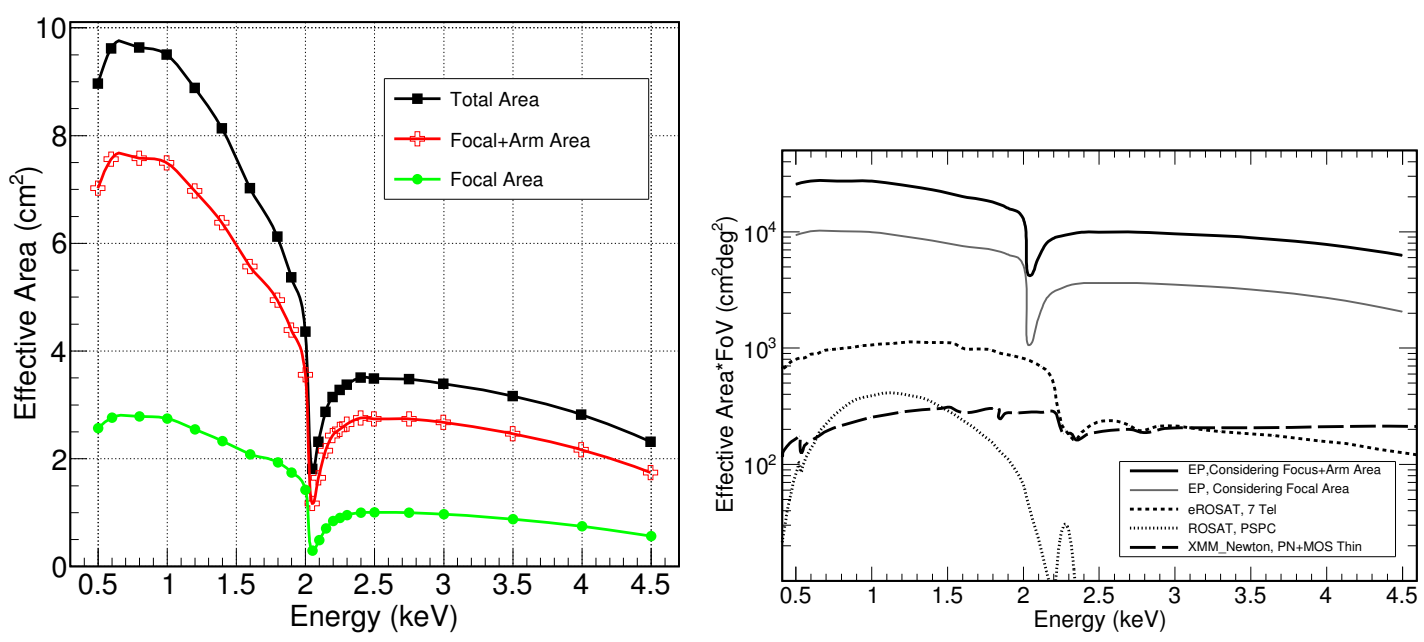

Figure 2: (left) Effective area curves for EP/WXT with GEM detectors, for the central focal spot (green), central plus the cruciform arms (red), and total (black; plus unfocused X-rays as diffuse background). The MPO arrays are coated with Iridium, and have surface roughness of $\sim 0.55 \mathrm{~nm}$ and the tilts of pores following a Gaussian distribution with $\sigma=0.85$ arcmin. The detector is filled with Xenon gas, and has a thin window of a $40 \mathrm{~nm}$-thick $\mathrm{Si}_{3} \mathrm{~N}_{4}$ foil coated with $30 \mathrm{~nm}$-thick Aluminum. (right) Grasp of WXT, in comparison with other focusing X-ray instruments. Figures adopted from Zhao et al. (2014) [17].

observations with FXT by targeting the new source within its FoV. Meanwhile, WXT continues to monitor the new sky region centering the position of the transient. It is essential to transmit alerts quickly to the ground, preferably within one minute or so. There are two possibilities currently under consideration: one is to make use of the French VHF ground station system by collaboration with CNES, and an alternative is to make use of the Chinese relay satellite network.

\section{Concluding remarks}

The X-ray Universe is rich in a large variety of energetic transients and variability, which are highly relevant to some of the key questions in astrophysics. The answers to these questions would have profound significance in understanding our transient Universe and its underlying physical laws, and would even revolutionise the relevant research. The Einstein Probe mission will attempt to address these questions by capturing faint flashes of X-ray radiation produced by energetic events within a cosmic horizon far beyond the reach of any current and previous missions. Its scientific impact will span a wide range of topics in astrophysics and fundamental physics, from stars, compact objects in our Galaxy and in nearby galaxies, black holes, supernovae, GRBs, galaxies to cosmology.

\section{Acknowledgments}

WY is grateful to many colleagues for valuable suggestions and discussion, and special thanks are given to M. Matsuoka, S. Komossa, N. Gehrels, M. Feroci, L. Piro, B. Zhang, B. Cordier, N. Kawai. The science consortium of Einstein Probe, composed of a large number of colleagues, is 


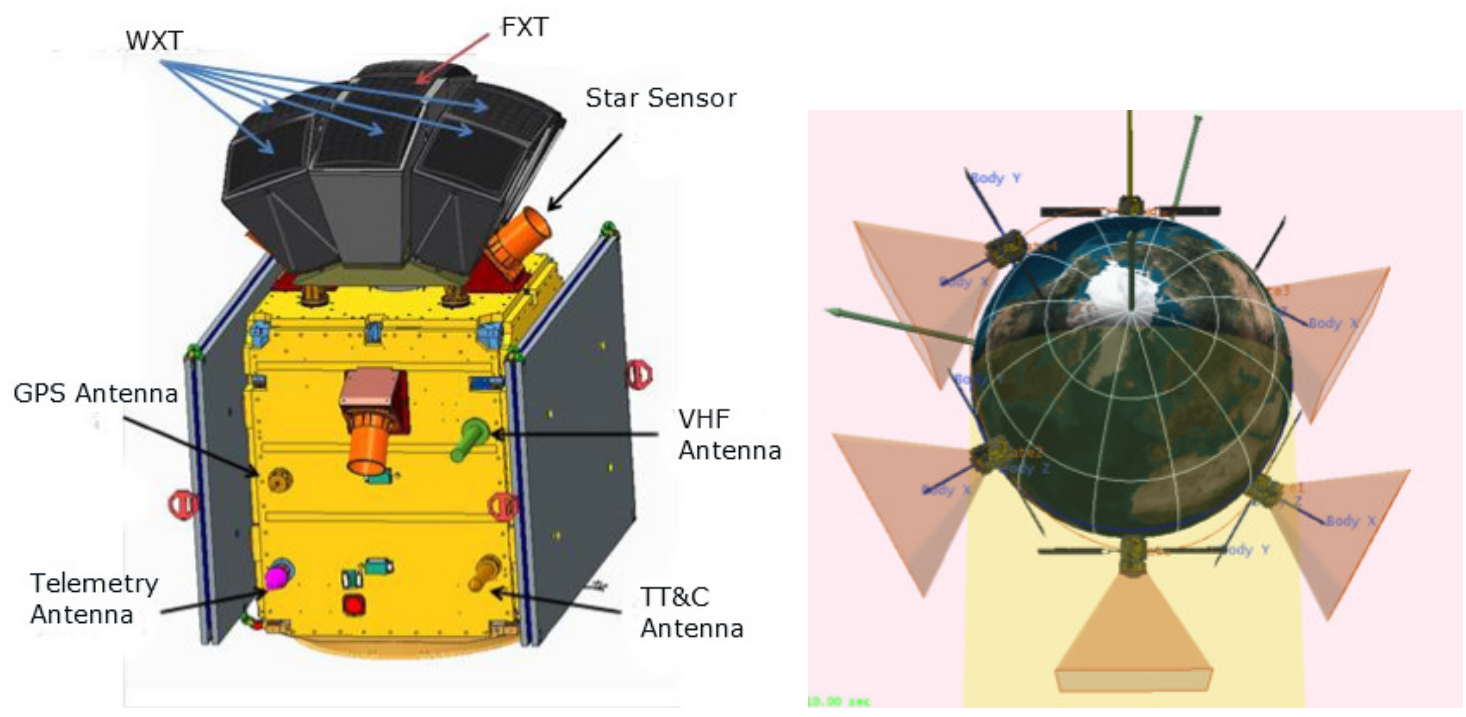

Figure 3: (left) Layout of the Einstein Probe satellite. (right) Illustration of the field-of-view and pointed observations in one orbit.

greatly acknowledged. This work is supported by the Strategic Priority Research Programme in Space Science of the CAS, Grant No. XDA04061100.

\section{References}

[1] N. Gehrels, et al., The Swift Gamma-Ray Burst Mission, ApJ 611 (2004) 1005

[2] N. Gehrels, Swift science and future, these proceedings (2015)

[3] W.B. Atwood, et al., The Large Area Telescope on the Fermi Gamma-Ray Space Telescope Mission, ApJ 697 (2009) 1071

[4] M. Matsuoka, et al., The MAXI Mission on the ISS: Science and Instruments for Monitoring All-Sky X-Ray Images, PASJ 61 (2009) 999

[5] M.J. Rees, Tidal disruption of stars by black holes of $10^{6}-10^{8}$ solar masses in nearby galaxies, Nature 333 (1988) 523

[6] S. Komossa \& N. Bade, The giant X-ray outbursts in NGC 5905 and IC 3599: Follow-up observations and outburst scenarios, A\&A 343 (1999) 775

[7] S. Komossa, Tidal disruption of stars by supermassive black holes: The X-ray view, EPJ Web of Conf., 39 (2012) id. 02001

[8] D. Burrows, et al., Relativistic jet activity from the tidal disruption of a star by a massive black hole, Nature 476 (2011) 421

[9] F.K. Liu, et al., A Milliparsec Supermassive Black Hole Binary Candidate in the Galaxy SDSS J120136.02+300305.5, ApJ 786 (2014) 103

[10] M. Kesden, Black-hole spin dependence in the light curves of tidal disruption events, PRD 86 (2012) 064026 
[11] J. Aasi, et al., Prospects for Localization of Gravitational Wave Transients by the Advanced LIGO and Advanced Virgo Observatories, arXiv/1304.0670 (2013)

[12] B. Schutz, Determining the Hubble constant from gravitational wave observations, Nature, $\mathbf{3 2 3}$ (1986) 310

[13] B. Zhang, Early X-Ray and Optical Afterglow of Gravitational Wave Bursts from Mergers of Binary Neutron Stars, ApJL 763 (2013) 22

[14] A.M. Soderberg, et al., An extremely luminous X-ray outburst at the birth of a supernova, Nature $\mathbf{4 5 3}$ (2008) 469

[15] J.R.P. Angel, Lobster eyes as X-ray telescopes, ApJ 233 (1979) 364

[16] R. Willingale, in preparation

[17] D. Zhao, et al., Ray tracing simulations for the wide-field $x$-ray telescope of the Einstein Probe mission based on Geant4 and XRTG4, SPIE, Proc. SPIE 9144, Space Telescopes and Instrumentation 2014: Ultraviolet to Gamma Ray 91444E (2014) 